\title{
ANALYTICITY OF ALMOST EVERYWHERE DIFFERENTIABLE FUNCTIONS
}

\author{
ERIC J. HOWARD
}

(Communicated by R. Daniel Mauldin)

\begin{abstract}
We develop a partitioning lemma (see Lemma 5) for superadditive set functions satisfying certain continuity conditions. This leads to a relatively simple proof of two theorems of A. S. Besicovitch on when a function of a complex variable that is continuous and differentiable outside of small exceptional sets is analytic (or almost everywhere equal to an analytic function).
\end{abstract}

\section{INTRODUCTION}

In 1931 A. S. Besicovitch proved the following two theorems concerning sufficient conditions for a function to be analytic (cf. [B]):

Theorem I. If a function $f(z)$ of a complex variable is defined and bounded in an open simply connected domain $D$ and is known to be differentiable at all points of $D$ except possibly at the points of a set $E$ of one-dimensional Hausdorff measure zero, then $f(z)$ is equal at each point of $D-E$ to a function analytic in all of $D$.

Theorem II. If a function $f(z)$ of a complex variable is defined and continuous in an open simply connected domain $D$ and is known to be differentiable at all points of $D$ except possibly at the points of a set $E$ of $\sigma$-finite one-dimensional Hausdorff measure, then $f(z)$ is analytic in all of $D$.

Two remarks about these theorems are in order. First, as Besicovitch noted in his paper, the exceptional sets in the two theorems cannot be enlarged if no additional conditions (e.g. topological conditions) are imposed on the sets. Second, as analyticity is a local property, it is clear that the simple connectivity of the domains in the two theorems is unnecessary.

The proof of Theorem I presented by Besicovitch involves a tedious induction with cumbersome inequalities, and takes considerable effort to understand fully. Not surprisingly, the proof of Theorem II, though similar to the first proof, is

Received by the editors September 13, 1989 and, in revised form, November 2, 1989.

1980 Mathematics Subject Classification (1985 Revision). Primary 28A10; Secondary 30B40.

Key words and phrases. $\delta$-fine partition, exceptional set, lower derivate.

This work originated from the Ph.D. thesis of Eric J. Howard written under the supervision of Washek F. Pfeffer at the University of California at Davis. 
even more tedious. In this paper we present a much simpler proof of these two theorems which bypasses the induction argument. We prove both results simultaneously, stating them as one theorem (Theorem 8).

Our purpose here is not to prove the most general form of Besicovitch's theorems. Instead, the emphasis is on a new proof of Besicovitch's results which relies on an important partitioning lemma (Lemma 5). The partitioning lemma is proved by exploiting the covering properties of dyadic squares and their relationship to Hausdorff measures developed by Besicovitch (Lemma 2), and is useful, not only for the results of this paper, but also for its application to the theory of integration. In particular, it may easily be employed to prove [P4, Lemma 3.8] (an outline of [P4] without proofs is given in [P5]) and [PY, Lemma 2.2] with the additive functions replaced by superadditive functions, thus allowing the additive majorants in the definitions of the variational integrals in $[\mathrm{P} 4, \S 4 ; \mathrm{PY}, \S 3]$ to be replaced by superadditive majorants. This in turn allows Riemann-type definitions of these integrals similar to that of [P3, Definition 3.1], in much the same way that the generalized Riemann integral is shown to be equivalent to a variational integral (see $[\mathrm{H}, \S 5])$.

In this paper, we use the partitioning lemma to prove a generalization to superadditive set functions in $\mathbf{R}^{m}$ of the fact that a differentiable function of one real variable is nondecreasing when its derivative is nonnegative (Theorem 7). The classical form of the theorem, that an additive function of compact intervals is nonnegative when its lower derivate is nonnegative, is well known (cf. [S, Chapter VI, Theorem (3.1) p. 190]). This result has been explored for superadditive functions in a more general topological setting in [P1, P2] and a closely related paper, [PW]. Here, we restrict our attention to superadditive functions of closed figures in $\mathbf{R}^{m}$. (Though it may seem more natural, in view of the present application of Lemma 5, to use subadditive functions and upper derivates, we choose to remain with superadditive functions and lower derivates, which are more appropriate for the applications mentioned in the previous paragraph.) From Theorem 7, Besicovitch's theorems follow using familiar techniques.

\section{Preliminaries}

We denote by $\mathbf{R}$ and $\mathbf{R}_{+}$the sets of real and positive real numbers, respectively. Throughout this paper, $m \geq 1$ is a fixed integer and, except where stated otherwise, all work is done in the $m$-dimensional space $\mathbf{R}^{m}$. For $x=$ $\left(\xi_{1}, \ldots, \xi_{m}\right)$ we let $|x|=\left(\sum_{i=1}^{m} \xi_{i}^{2}\right)^{1 / 2}$ and $\|x\|=\max \left\{\left|\xi_{1}\right|, \ldots,\left|\xi_{m}\right|\right\}$. If $A \subset \mathbf{R}^{m}$ and $x \in \mathbf{R}^{m}$, we let $d(A)$ and $\operatorname{dist}(x, A)$ denote the diameter of $A$ and the distance between $x$ and $A$, both with respect to the norm $\|x\|$. If $\delta$ is a positive number then $U(A, \delta)=\left\{y \in \mathbf{R}^{m}: \operatorname{dist}(y, A)<\delta\right\}$, but we write $U(x, \delta)$ in place of $U(\{x\}, \delta)$. The closure, interior, boundary, and $m$ dimensional Lebesgue measure of $A$ are denoted by $A^{-}, A^{\circ}, \partial A$, and $|A|$, respectively. 
In this paper, an interval is the cartesian product of $m$ nondegenerate compact intervals in $\mathbf{R}$, and a figure is a finite union of intervals. Thus, intervals and figures are always closed. For any set $A \subset \mathbf{R}^{m}$, we let $\mathscr{F}(A)$ denote the collection of all figures which are subsets of $A$, together with the empty set. As the collection $\mathscr{F}(A)$ is not closed with respect to set difference and intersection, we define two corresponding set operations under which $\mathscr{F}(A)$ is closed; namely, $B_{1} \ominus B_{2}=\left(B_{1}-B_{2}\right)^{-}$and $B_{1} \odot B_{2}=\left[\left(B_{1} \cap B_{2}\right)^{\circ}\right]^{-}$. Two figures $B_{1}$ and $B_{2}$ are called nonoverlapping if $B_{1} \odot B_{2}=\varnothing$. An interval is called a cube if it is the cartesian product of $m$ intervals in $\mathbf{R}$ of equal length, and a dyadic cube is a cube of the form $\prod_{i=1}^{m}\left[k_{i} 2^{-n},\left(k_{i}+1\right) 2^{-n}\right]$ where $n, k_{1}, \ldots, k_{m}$ are integers and $n \geq 0$. The terms rectangle and square refer to intervals and cubes, respectively, in $\mathbf{R}^{2}$. In particular, rectangles and squares are always closed and have sides parallel to the coordinate axes.

Let $A$ be a figure and let $E \subset A$. A partition in $A \bmod E$ is a collection $\mathscr{P}=$ $\left\{\left(A_{1}, x_{1}\right), \ldots,\left(A_{p}, x_{p}\right)\right\}$ where $\left\{A_{1}, \ldots, A_{p}\right\}$ is a family of nonoverlapping subintervals of $A$ and $x_{i} \in A_{i}-E$ for $i=1, \ldots, p$. We write $\cup \mathscr{P}=$ $\bigcup\{B:(B, x) \in \mathscr{P}\}$. It should be noted that we refer to a partition in $A$ (as opposed to of $A$ ) since $\bigcup \mathscr{P}$ need not equal $A$. The partition $\mathscr{P}$ is called dyadic if each $A_{i}$ is a dyadic cube, and if $\delta: A-E \rightarrow \mathbf{R}_{+}$, then $\mathscr{P}$ is called $\delta$-fine when $d\left(A_{i}\right)<\delta\left(x_{i}\right)$ for $i=1, \ldots, p$. The following lemma is a dyadic version of Cousin's lemma.

Lemma 1. If $A$ is a dyadic cube and $\delta: A \rightarrow \mathbf{R}_{+}$, then there is a $\delta$-fine dyadic partition $\mathscr{P}$ in $A \bmod \varnothing$ with $A=\bigcup \mathscr{P}$.

Proof. Assume the lemma is not true. Divide $A$ into $2^{m}$ dyadic cubes $A^{(1)}, \ldots$, $A^{(2 m)}$ with $d\left(A^{(i)}\right)=d(A) / 2$. Since $A$ does not have a $\delta$-fine dyadic partition, at least one of the $2^{m}$ subcubes does not have a $\delta$-fine dyadic partition; call it $A_{1}$. Applying the same reasoning to $A_{1}$ and continuing in this manner, we obtain a sequence $\left\{A_{n}\right\}$ of nested dyadic cubes, each of which has no $\delta$-fine dyadic partition and $d\left(A_{n}\right) \rightarrow 0$. Letting $\bigcap_{n} A_{n}=\{x\}$ we eventually have $d\left(A_{n}\right)<\delta(x)$ for large $n$. Thus, $\left\{\left(A_{n}, x\right)\right\}$ is a $\delta$-fine dyadic partition of $A_{n}$ for large $n$, which is a contradiction and the lemma is proved.

If $A$ is a figure, we let $\|A\|$ denote the usual $(m-1)$-dimensional surface area. For a set $E \subset \mathbf{R}^{m}$, we denote by $\mathscr{H}(E)$ the $(m-1)$-dimensional outer Hausdorff measure of $E$ defined as in $[\mathrm{Fe}, \S 2.10 .2$, p. 171] so that $\mathscr{H}(\partial A)=$ $\|A\|$ for each figure $A$. We note that $\mathscr{H}$ differs from $\mathscr{H}^{m-1}$ defined in [Fa, $\S 1.2$, p. 7] by a multiplicative constant (cf. [Fa, Theorem 1.12, p. 13]). As in [P4], we call a set slight if $\mathscr{H}(E)=0$ and thin if it has $\sigma$-finite $\mathscr{H}$-measure. The slight and thin sets defined in this way are larger than those of [P3, PY]; in particular, they are not necessarily compact. The next lemma is the basic tool used in the proof of Lemma 5, and follows from [Fa, Theorem 5.1, p. 65].

Lemma 2. There is a constant $\kappa>0$ which depends only on $m$ and has the following property: if $E \subset \mathbf{R}^{m}$ and $\mathscr{H}(E)<a$, then for each $\eta>0$ we can find 
a nonoverlapping sequence $\left\{B_{n}\right\}$ of dyadic cubes with diameters less than $\eta$ so that $E \subset\left(\bigcup B_{n}\right)^{\circ}$ and $\sum\left[d\left(B_{n}\right)\right]^{m-1}<\kappa a$.

The fact that the sequence $\left\{B_{n}\right\}$ in Lemma 2 can be taken to be nonoverlapping is a consequence of the fact that any collection of dyadic cubes has a nonoverlapping subcollection with the same union. This observation plays a critical role in the proof of Lemma 5.

\section{FUNCTIONS OF FIGURES}

By a function on $\mathscr{F}(A)$ we mean a function which assigns a real value to each element of $\mathscr{F}(A)$. The following definition parallels [P4, Definitions 3.1 and 3.6].

Definition 3. Let $A$ be a figure and let $F$ be a function on $\mathscr{F}(A)$. We say that $F$ is:

(i) lower bounded if given $\varepsilon>0$ there is a $\delta>0$ such that $F(B)>-\varepsilon$ for each $B \in \mathscr{F}(A)$ with $\|B\|<\delta$;

(ii) lower continuous in a set $E \subset A$ if given $\varepsilon>0$, there is a $\delta>0$ such that $F(B)>-\varepsilon$ for each $B \in \mathscr{F}(A)$ with $B \subset A \cap U(E, \delta),|B|<\delta$, and $\|B\|<1 / \varepsilon$; and

(iii) lower amiable if it is lower bounded, and if there is a slight set $S \subset A$ such that $F$ is lower continuous in each compact set $E \subset A-S$.

If both $F$ and $-F$ are lower bounded, lower continuous, or lower amiable, we say that $F$ is bounded, continuous, or amiable, respectively.

Example 4. Identify the complex plane with $\mathbf{R}^{2}$ and let $f(z)$ be a function of a complex variable in a figure $A \subset \mathbf{R}^{2}$. Define $F(\varnothing)=0$ and $F(B)=$ $\left|\int_{\partial B} f(z) d z\right|$ for every figure $B \subset A$, where we adhere to the usual convention that the boundary of $B$ is oriented counterclockwise. We prove the following two facts:

(F1) If $f$ is bounded in $A$ then $F$ is bounded in $A$.

(F2) If $f$ is continuous in a compact set $E \subset A$ then $F$ is continuous in $E$.

To prove (F1), simply note that if $K$ is a bound for $|f(z)|$ and $B$ is any figure in $A$, then $\left|\int_{\partial B} f(z) d z\right| \leq \int_{\partial B}|f(z)||d z| \leq K\|B\|$ so $F(B) \rightarrow 0$ as $\|B\| \rightarrow 0$.

The proof of (F2) takes more work. Choose $\varepsilon>0$ and let $f(z)=u(x, y)+$ $i v(x, y)$. Since $u$ and $v$ are continuous in the compact set $E$, by the StoneWeierstrass theorem there are polynomials $p(x, y)$ and $q(x, y)$ such that if $g(z)=p(x, y)+i q(x, y)$ then $|f(z)-g(z)|<\varepsilon^{2} / 6$ for each $z \in E$. For every $z \in E$, find $\delta_{z}>0$ so that $|f(z)-f(w)|<\varepsilon^{2} / 6$ and $|g(z)-g(w)|<\varepsilon^{2} / 6$ whenever $w \in A \cap U\left(z, \delta_{z}\right)$. Since $E$ is compact there is a $\delta>0$ such that $U(E, \delta) \subset \bigcup_{z \in E} U\left(z, \delta_{z}\right)$. Hence, if $w \in U(E, \delta)$ then $w \in U\left(z, \delta_{z}\right)$ for some $z \in E$ and

$$
|f(w)-g(w)| \leq|f(w)-f(z)|+|f(z)-g(z)|+|g(z)-g(w)|<\varepsilon^{2} / 2 .
$$


Let $M=2 \max _{z \in A}|\partial g / \partial \bar{z}|$ where $\partial g / \partial \bar{z}=(\partial g / \partial x+i \partial g / \partial y) / 2$, and decrease $\delta$ if necessary so that $M \delta<\varepsilon / 2$. If $B \subset A \cup U(E, \delta)$ is a figure with $|B|<\delta$ and $\|B\|<1 / \varepsilon$, then by the complex form of Green's theorem,

$$
\left|\int_{\partial B} g(z) d z\right|=\left|2 i \iint_{B} \frac{\partial g}{\partial \bar{z}} d x d y\right| \leq 2 \frac{M}{2}|B|<M \delta<\frac{\varepsilon}{2} .
$$

Therefore, we see that

$$
\left|\int_{\partial B} f(z) d z\right| \leq \int_{\partial B}|f(z)-g(z)||d z|+\left|\int_{\partial B} g(z) d z\right| \leq \frac{\varepsilon^{2}}{2}\|B\|+\frac{\varepsilon}{2}<\varepsilon .
$$

That is, $|F(B)|<\varepsilon$ so $F$ is continuous in $E$.

Remark. If $f(z)$ is bounded in $A$ and continuous in $A-S$ for some slight set $S$, then (F1) and (F2) show that $F$ is amiable in $A$.

\section{THE PARTITIONING LEMMA FOR SUPERADDITIVE FUNCTIONS}

For a figure $A$, a function on $\mathscr{F}(A)$ is superadditive if $F\left(\bigcup_{D \in \mathscr{D}} D\right) \geq$ $\sum_{D \in \mathscr{D}} F(D)$ for each finite nonoverlapping family $\mathscr{D}$ in $\mathscr{F}(A)$.

Remark. For any superadditive function $F$, the inequality $F(B) \geq F(B)+$ $F(\varnothing)$ shows that $F(\varnothing) \leq 0$. If $F$ is also lower bounded (and hence, if $F$ is lower amiable), then $F(\varnothing)=0$.

Lemma 5. Let $A$ be a dyadic cube and let $F$ be a lower amiable superadditive function on $\mathscr{F}(A)$. If $T$ is any thin set, then for $\varepsilon>0$ and $\delta: A-T \rightarrow \mathbf{R}_{+}$ there is a $\delta$-fine dyadic partition $\mathscr{P}$ in $A$ mod $T$ such that $F(A \ominus \bigcup \mathscr{P})>-\varepsilon$. Proof. Since $F$ is lower bounded, there is an $\eta>0$ such that $F(B)>-\varepsilon / 2$ for each figure $B \subset A$ with $\|B\|<\eta$. Let $S$ be a slight set such that $F$ is lower continuous in each compact subset of $A-S$. By Lemma 2 there is a sequence $\left\{S_{n}\right\}$ of nonoverlapping dyadic cubes such that $S \subset G=\left(\bigcup S_{n}\right)^{\circ}$ and

$$
\sum_{n}\left[d\left(S_{n}\right)\right]^{m-1}<\frac{\eta}{2 m}
$$

Since $T^{\prime}=T-G$ is thin, there is a disjoint sequence $\left\{T_{i}\right\}$ of sets with $T^{\prime}=$ $\cup T_{i}$ and $\mathscr{H}\left(T_{i}\right)<1$ for $i=1,2, \ldots$. For each $i$, choose $\varepsilon_{i}>0$ with $\varepsilon_{i}<\min \left\{1,1 / 2 m \kappa, \varepsilon 2^{-i}\right\}$ where $\kappa$ is the constant from Lemma 2. By the lower continuity of $F$ in $A-G$, there are positive numbers $\eta_{i}$ such that $F(B)>-\varepsilon_{i} / 2$ for each figure $B \subset A \cap U\left(A-G, \eta_{i}\right)$ with $|B|<\eta_{i}$ and $\|B\|<2 / \varepsilon_{i}$. Applying Lemma 2 to each $T_{i}$ we obtain sequences $\left\{T_{i, n}\right\}_{n}$ of nonoverlapping dyadic cubes with diameters less than $\eta_{i} \varepsilon_{i}$ such that $T_{i} \subset$ $\left(\bigcup_{n} T_{i, n}\right)^{\circ}$ and

$$
\sum_{n}\left[d\left(T_{i, n}\right)\right]^{m-1}<\kappa .
$$

We may assume that $T_{i}$ intersects $T_{i, n}$ for each $n$, so $T_{i, n} \subset U\left(A-G, \eta_{i}\right)$. Let $\mathscr{U}$ be a nonoverlapping subcollection of $\left\{S_{n}\right\} \cup\left\{T_{i, n}: i, n=1,2, \ldots\right\}$ 
with $\bigcup \mathscr{U}=\bigcup S_{n} \cup\left(\bigcup_{i, n} T_{i, n}\right)$ so $S \cup T \subset(\bigcup \mathscr{U})^{\circ}$. Choose a function $\hat{\delta}: A \rightarrow$ $\mathbf{R}_{+}$with $\hat{\delta}(x) \leq \delta(x)$ on $A-T$ which satisfies the following condition: if $x \in S \cup T$ and $B$ is a dyadic cube containing $x$ with $d(B)<\hat{\delta}(x)$, then $B \subset U$ for some $U \in \mathscr{U}$. By Lemma 1 , there is a $\hat{\delta}$-fine partition $\mathscr{Q}$ of $A$ such that $A=\bigcup \mathscr{Q}$. Let $\mathscr{V}$ be the collection of all cubes in $\mathscr{U}$ which contain a cube from $\mathscr{Q}$, and let $\mathscr{P}=\left\{\left(A_{1}, x_{1}\right), \ldots,\left(A_{p}, x_{p}\right)\right\}$ be the collection of all $(B, x) \in \mathscr{Q}$ such that $B$ is not contained in a cube from $\mathscr{U}$. Since for any two overlapping dyadic cubes, one must contain the other, $A_{j}$ does not overlap $\cup \mathscr{V}$ for each $j=1, \ldots, p$. Furthermore, each cube from $\mathscr{Q}$ is either contained in $\bigcup \mathscr{V}$ or it is not, so $A \ominus \bigcup \mathscr{P}=\bigcup \mathscr{V}$. By the way that $\hat{\delta}(x)$ was chosen on $T$, we have $x_{j} \in A-T$ for each $j=1, \ldots, p$, so $\mathscr{P}$ is a $\delta$-fine partition in $A$ mod $T$. Separate $\mathscr{V}$ into the following disjoint finite subcollections:

$$
\mathscr{S}=\mathscr{V} \cap\left\{S_{n}\right\}, \quad \mathscr{T}_{1}=\left(\mathscr{V} \cap\left\{T_{1, n}: n=1,2, \ldots\right\}\right)-\mathscr{S}
$$

and for $i=2,3, \ldots$,

$$
\mathscr{T}_{i}=\left(\mathscr{V} \cap\left\{T_{i, n}: n=1,2, \ldots\right\}\right)-\left(\mathscr{S} \cup\left(\bigcup_{j=1}^{i-1} \mathscr{T}_{j}\right)\right) .
$$

Since $\mathscr{V}$ is finite, there is an integer $r$ such that $\mathscr{V}=\mathscr{S} \cup\left(\bigcup_{i=1}^{r} \mathscr{T}_{i}\right)$. By (1),

$$
\left\|\bigcup_{B \in \mathscr{S}} B\right\| \leq \sum_{B \in \mathscr{S}}\|B\| \leq \sum_{n}\left\|S_{n}\right\| \leq 2 m \sum_{n}\left[d\left(S_{n}\right)\right]^{m-1}<2 m \frac{\eta}{2 m}=\eta,
$$

so $F\left(\bigcup_{B \in \mathscr{S}} B\right)>-\varepsilon / 2$. By $(2)$,

$$
\left\|\bigcup_{B \in \mathscr{T}_{i}} B\right\| \leq \sum_{B \in \mathscr{T}_{i}}\|B\| \leq \sum_{n}\left\|T_{i, n}\right\| \leq 2 m \sum_{n}\left[d\left(T_{i, n}\right)\right]^{m-1}<2 m \kappa<\frac{1}{\varepsilon_{i}}<\frac{2}{\varepsilon_{i}},
$$

from which follows

$$
\left|\bigcup_{B \in \mathscr{T}_{i}} B\right|=\sum_{B \in \mathscr{T}_{i}}|B| \leq \eta_{i} \varepsilon_{i} \sum_{B \in \mathscr{T}_{i}}\|B\|<\eta_{i}
$$

Hence, $F\left(\bigcup_{B \in \mathscr{T}_{i}} B\right)>-\varepsilon_{i} / 2$ so

$$
\begin{aligned}
F(A \ominus \bigcup \mathscr{P})=F\left(\bigcup_{B \in \mathscr{V}} B\right) & \geq F\left(\bigcup_{B \in \mathscr{S}} B\right)+\sum_{i=1}^{r} F\left(\bigcup_{B \in \mathscr{S}_{i}} B\right) \\
& >-\frac{\varepsilon}{2}-\sum_{i=1}^{r} \frac{\varepsilon_{i}}{2}>-\varepsilon .
\end{aligned}
$$

The next lemma generalizes Lemma 5 by replacing the dyadic cube $A$ by an arbitrary figure. It is worth noting that the same proof can be used to replace $A$ by any bounded set with thin boundary, but we do not need such generality here. 
Lemma 6. Let $A$ be a figure and let $F$ be a lower amiable superadditive function on $\mathscr{F}(A)$. If $T$ is any thin set, then for $\varepsilon>0$ and $\delta: A-T \rightarrow \mathbf{R}_{+}$there is a $\delta$-fine dyadic partition $\mathscr{P}$ in $A \bmod T$ such that $F(A \ominus \bigcup \mathscr{P})>-\varepsilon$.

Proof. Let $\left\{C_{1}, \ldots, C_{s}\right\}$ be a nonoverlapping collection of dyadic cubes such that $A \subset C=\bigcup C_{i}$. Extend $F$ to a superadditive lower amiable function $\hat{F}$ on $\mathscr{F}(C)$ by setting $\hat{F}(B)=F(B \odot A)$ for each $B \in \mathscr{F}(C)$. Choose $\hat{\delta}: C-(T \cup \partial A) \rightarrow \mathbf{R}_{+}$such that $\hat{\delta}(x) \leq \delta(x)$ for each $x \in A-T$ and $\hat{\delta}(x)<\operatorname{dist}(x, \partial A)$ for each $x \in C-(T \cup \partial A)$. By Lemma 5, for each $i=1, \ldots, s$, there is a $\hat{\delta}$-fine dyadic partition $\mathscr{P}_{i}$ in $C_{i} \bmod T \cup \partial A$ such that $\hat{F}\left(C_{i} \ominus P_{i}\right)>-\varepsilon / s$ where $P_{i}=\bigcup\left\{B:(B, x) \in \mathscr{P}_{i}\right\}$. Since $\hat{\delta}(x) \leq \operatorname{dist}(x, \partial A)$ for $x \in C-(T \cup \partial A)$, if $(B, x) \in \bigcup_{i=1}^{s} \mathscr{P}_{i}$ then either $B \subset A$ or $B \cap A=\varnothing$. Let $\mathscr{P}=\left\{\left(A_{1}, x_{1}\right), \ldots,\left(A_{q}, x_{q}\right)\right\}$ be all pairs $(B, x) \in \bigcup_{i=1}^{s} \mathscr{P}_{i}$ such that $B \subset A^{\circ}$. Then since $\hat{\delta}(x) \leq \delta(x)$ on $A^{\circ}-T$, the partition $\mathscr{P}$ is $\delta$-fine in $A$ $\bmod T$ and

$$
\begin{aligned}
F(A \ominus \bigcup \mathscr{P}) & =F\left(A \cap \bigcup_{i=1}^{s}\left[C_{i} \ominus P_{i}\right]\right)=\hat{F}\left(\bigcup_{i=1}^{s}\left[C_{i} \ominus P_{i}\right]\right) \\
& \geq \sum_{i=1}^{s} \hat{F}\left(C_{i} \ominus P_{i}\right)>-\sum_{i=1}^{s} \frac{\varepsilon}{s}=-\varepsilon .
\end{aligned}
$$

\section{LOWER DERIVATES AND THE THEOREMS OF BESICOVITCH}

Let $A$ be a figure and let $F$ be a function on $\mathscr{F}(A)$. The lower derivate of $F$ at a point $x \in A^{\circ}$ is defined to be

$$
D_{*} F(x)=\inf \liminf _{n} \frac{F\left(C_{n}\right)}{\left|C_{n}\right|},
$$

where the infimum is taken over all sequences $\left\{C_{n}\right\}$ of closed subcubes (not necessarily dyadic) of $A$ such that $x \in C_{n}$ and $\lim d\left(C_{n}\right)=0$.

Theorem 7. Let $A$ be a figure and let $F$ be a lower amiable superadditive function on $\mathscr{F}(A)$. If there is a thin set $T$ such that $D_{*} F(x) \geq 0$ for each $x \in A^{\circ}-T$, then $F$ is nonnegative.

Proof. As the restriction of $F$ to $\mathscr{F}(B)$ is a lower amiable superadditive function on $\mathscr{F}(B)$ for each $B \in \mathscr{F}(A)$, it suffices to show that $F(A) \geq 0$. Choose $\varepsilon>0$. Since each $x \in \mathbf{R}^{m}$ is in only countably many closed dyadic cubes, for $x \in A^{\circ}-T$ there is a $\delta(x)>0$ such that $F(C) /|C|>-\varepsilon /(2|A|)$ for each dyadic cube $C$ with $x \in C$ and $d(C)<\delta(x)$. Thus, we have a map $\delta: A-(T \cup \partial A) \rightarrow \mathbf{R}_{+}$and by Lemma 6 , there is a $\delta$-fine dyadic partition $\left\{\left(A_{1}, x_{1}\right), \ldots,\left(A_{p}, x_{p}\right)\right\}$ in $A \bmod T \cup \partial A$ such that $F\left(A \ominus \bigcup A_{i}\right)>-\varepsilon / 2$. Therefore,

$$
F(A) \geq F\left(A \ominus \bigcup A_{i}\right)+\sum_{i=1}^{p} F\left(A_{i}\right)>-\frac{\varepsilon}{2}-\sum_{i=1}^{p} \frac{\varepsilon\left|A_{i}\right|}{2|A|} \geq-\varepsilon .
$$

Since $\varepsilon$ was arbitrary, $F(A) \geq 0$. 
It is interesting to note that the condition $D_{*} F(x) \geq 0$ is more than is needed. It would be sufficient to assume that $D_{d} F(x) \geq 0$ on $A^{\circ}-T$ where $D_{d} F(x)$ is the "lower dyadic derivate" $\lim \inf F\left(C_{n}\right) /\left|C_{n}\right|$, the sequence $\left\{C_{n}\right\}$ being the collection of dyadic cubes containing $x$ in a decreasing order.

The next theorem contains the two theorems of Besicovitch quoted in the introduction of this paper.

Theorem 8. Let $D$ be an open set in the plane and let $S$ and $T$ be slight and thin sets, respectively. If $f(z)$ is a function of a complex variable in $D$ which is bounded on each compact subset of $D$, is continuous at each point of $D-S$, and has a complex derivative at each point of $D-T$, then $f$ is equal at each point of $D-S$ to a function which is analytic in $D$.

To prove Theorem 8 we first show in Lemma 9 that $\int_{\partial R} f(z) d z=0$ for each rectangle $R \subset D$, but as the function $f$ is not continuous, Morera's theorem is not sufficient to finish the proof.

Lemma 9. Let $D, S, T$, and $f$ be as in Theorem 8. Then $\int_{\partial R} f(z) d z=0$ for each rectangle $R \subset D$.

Proof. Let $R$ be a rectangle in $D$ and define $F(\varnothing)=0$ and

$$
F(B)=-\left|\int_{\partial B} f(z) d z\right|
$$

for each figure $B \subset R$. Clearly, $F$ is superadditive and by Example $4, F$ is amiable. Choose $z \in R-T$. Since $f^{\prime}(z)$ exists, we can write

$$
f(\zeta)=f(z)+f^{\prime}(z)(\zeta-z)+\phi_{z}(\zeta)(\zeta-z),
$$

where $\phi_{z}(\zeta) \rightarrow 0$ as $\zeta \rightarrow z$. Choose $\varepsilon>0$ and let $\delta>0$ be such that $\left|\phi_{z}(\zeta)\right|<\varepsilon / 4 \sqrt{2}$ when $|\zeta-z|<\delta$. Then for any square $C \subset R$ with $z \in C$ and $d(C)<\delta / \sqrt{2}$,

$$
\begin{aligned}
\left|\int_{\partial C} f(\zeta) d \zeta\right| & =\left|\int_{\partial C} \phi_{z}(\zeta)(\zeta-z) d \zeta\right| \leq \int_{\partial C}\left|\phi_{z}(\zeta)\right||\zeta-z||d \zeta| \\
& \leq \frac{\varepsilon}{4 \sqrt{2}} \sqrt{2} d(C)\|C\|=\varepsilon|C| .
\end{aligned}
$$

This implies that $F(C) /|C| \geq-\varepsilon$ and since $\varepsilon$ was arbitrary, $D_{*} F(z) \geq 0$. By Theorem 7 we see that $F(R) \geq 0$ but $F$ is nonpositive so $F(R)=0$. That is, $\int_{\partial R} f(z) d z=0$.

Proof of Theorem 8. Choose an open square $V$ in $D$ with $V^{-} \subset D$. Call a path $\gamma$ in $V$ admissible if it consists of a finite number of line segments parallel to the axes and let $l(\gamma)$ denote its length. Fix $z_{0} \in V$ and for each $z$ in $V$ define $g(z)=\int_{\gamma} f(\zeta) d \zeta$ where $\gamma$ is any admissible path in $V$ from $z_{0}$ to $z$. Since $S$ is slight, the integral always exists and by Lemma 9, the function $g$ is well defined. It is easy to show that the boundedness of $f$ implies that $g$ is 
continuous. Moreover, if $z$ and $w$ are points of $V$ and $\gamma$ is an admissible path from $z$ to $w$ then

$$
|[g(w)-g(z)]-f(z)(w-z)|=\left|\int_{\gamma}[f(\zeta)-f(z)] d \zeta\right| \leq l(\gamma) \max _{\zeta \in \gamma}|f(\zeta)-f(z)| \text {. }
$$

Since $\gamma$ can be chosen so that $l(\gamma) \leq 2|w-z|$, then $g$ has a complex derivative equal to $f(z)$ at each point $z \in V$ where $f$ is continuous. Thus, by Lemma 9 , $\int_{\partial R} g(z) d z=0$ for each rectangle $R$ in $V$ so by Morera's theorem, $g$ is analytic. Hence, $f$ is equal to the analytic $g^{\prime}$ at each point of $V$ where $f$ is continuous. As $V$ was arbitrary, the proof is complete.

This last proof is essentially a proof of a simple generalization of Morera's theorem to functions whose set of discontinuities is a slight set. It should be noted that much more general versions of Morera's theorem exist (see [R; $Z$, Theorem 1]) which could be applied to immediately deduce Theorem 8 from Lemma 9. The above elementary argument was presented in order to be consistent with our goal of presenting a simple proof of Besicovitch's theorems.

Finally, by using standard techniques, the differentiability requirement in Theorem 8 can be relaxed to that of [S, Chapter VI, Theorem (5.3), p. 197].

\section{REFERENCES}

[B] A. S. Besicovitch, On sufficient conditions for a function to be analytic, and behaviour of analytic functions in the neighborhood of non-isolated singular points, Proc. London Math. Soc. 32 (1931), 1-9.

[Fa] K. J. Falconer, The geometry of fractal sets, Cambridge Univ. Press, Cambridge, 1985.

[Fe] H. Federer, Geometric measure theory, Springer-Verlag, New York, 1969.

[H] R. Henstock, Lectures on the theory of integration, World Scientific, Singapore, 1988.

[P1] W. F. Pfeffer, A note on the lower derivate of a set function and semihereditary systems of sets, Proc. Amer. Math. Soc. 18 (1967), 1020-1025.

[P2] _ On the lower derivate of a set function, Canad. J. Math. 20 (1968), 1489-1498.

[P3] _ The multidimensional fundamental theorem of calculus, J. Australian Math. Soc. 43 (1987), 143-170.

[P4] _ Divergence theorem for vector fields with singularities, unpublished.

[P5] _ Stokes theorem for forms with singularities, C. R. Acad. Sci. Paris, Sér. I 306 (1988), 589-592.

[PW] W. F. Pfeffer and W. J. Wilber, A note on cluster points of a semihereditary stable system of sets, Proc. Amer. Math. Soc. 21 (1969), 121-125.

[PY] W. F. Pfeffer and Wei-Chi Yang, A multidimensional variational integral and its extensions, Real Analysis Exchange (1) 15 (1989-90), 111-169.

[R] H. L. Royden, A generalization of Morera's theorem, Ann. Polon. Math. 12 (1962), 199-202.

[S] S. Saks, Theory of the integral, Dover, New York, 1964.

[Z] L. Zalcman, Analyticity and the Pompeiu problem, Arch. Rational Mech. Anal. 47 (1972), 237-254.

Department of Mathematics, University of California, Davis, California 95616 